\title{
Uncertain Resource-Constrained Project Scheduling Problem with Net Present Value Criterion
}

\author{
Chenkai Zhao ${ }^{1}$, Hua Ke ${ }^{1 *}$ and Zhiyi Chen ${ }^{2}$
}

\section{${ }^{*}$ Correspondence:}

hke@tongji.edu.cn

'School of Economics and

Management, Tongji University,

Siping Road, Shanghai 200092,

China

Full list of author information is

available at the end of the article

\begin{abstract}
On the basis of uncertainty theory, plenty of researches have been done on uncertain resource-constrained project scheduling problems. Instead of minimizing the makespan, in this paper, we address the maximization of net present value of a project's cash flows when activity durations are assumed to be uncertain. In addition to precedence constraint and resource constraint involved in resource-constrained project scheduling problem, a deadline constraint is taken into account. Thus, our aim is to maximize net present value and to satisfy the deadline constraint as well. Accordingly, we introduce three models and utilize a revised estimation of distribution algorithm to solve this problem. This work may provide net present value criterion for financial officers on project scheduling.
\end{abstract}

Keywords: Project scheduling, Uncertainty theory, Net present value, Estimation of distribution algorithm

\section{Introduction}

Project scheduling is to assign activity starting times based on scheduling objectives [1]. Researches in this area have primarily emphasized on modeling and algorithmic developments for specific classes of project scheduling problems, such as net present value (NPV) maximization, quality maximization, and makespan minimization [2]. Most literature on project scheduling focused on arranging activity starting times in such a way that the project makespan is minimized. This objective may be unsuitable for capitalintensive IT and construction projects, where large amounts of money are invested over a long period of time. In such an environment, the wise coordination of cash flows crucially affects the profitability of a project. This suggests that in such situations, financial aspects should be the center of decision makers' attention. The financial benefit of a project is measured by its NPV, which is determined by discounting all arising cash flows to the start time of the project. In other words, NPV can be regarded as the cash equivalent of undertaking the project. In reality, contractors sign contracts with clients to define ways of receiving payments linked with progress of related activities. Thus, different contracts lead to different time points of payments and cash flows vary correspondingly. In this paper, we assume that contractors only receive payments when related activities are completed. For instance, when a construction company undertakes a project consisting

(c) The Author(s). 2016 Open Access This article is distributed under the terms of the Creative Commons Attribution 4.0 International License (http://creativecommons.org/licenses/by/4.0/), which permits unrestricted use, distribution, and reproduction in any medium, provided you give appropriate credit to the original author(s) and the source, provide a link to the Creative Commons license, and indicate if changes were made. 
of lots of activities funded by its client, it seems unreasonable to make payments happen either at the beginning of the project or at the end of the project. Therefore, to avoid being cheated by each other and to keep the project progress on schedule, an agreement is signed to set payments on the completion time of each activity. This criterion can be applied to resource-constrained project scheduling problem (RCPSP), discrete time-cost trade-off problem (TCTP), and other classical subproblems of project scheduling problems. Scheduling projects to maximize NPV in a deterministic setting has been studied under a broad range of contractual arrangements and planning constraints [3], but in practice, there are frequently significant indeterminacies. In this paper, we focus on an uncertain resource-constrained project scheduling problem with discounted cash flows (URCPSPDC) additionally considering a due date constraint.

Then, we propose three uncertain models. The chance-constrained model is proposed to maximize NPV with a certain belief degree, which can be applied to decision makers who may be risk-averse and desire to realize the objective value with a pretty high belief degree. Besides, the expected value model aims at maximizing the expected NPV of cash flow received on the completion time of each activity. The chance maximization model is based on dependent-chance programming. Moreover, a revised estimation of distribution algorithm (EDA) is utilized in this paper. EDAs have been developed for years and successfully applied in RCPSP. For more details about EDA for RCPSP, readers may refer to $[4,5]$.

The remainder of this paper is as follows. In Section Literature Review, we summarize related literature. Section Preliminaries introduces some basic concepts in uncertainty theory. Section NPV Models describes URCPSPDC in detail and proposes three corresponding NPV models to satisfy the demand of financial officers. To solve these three models, revised EDAs are designed in Section Revised EDA. Section Numerical Examples conducts some numerical examples. Finally, conclusions are drawn in Section Conclusion and Future Work.

\section{Literature Review}

Maximizing the NPV of a project was first suggested by Russell [6]. Besides, Buss and Rosenblatt [7] as well as Sobel et al. [8] extended Russell's model by including indeterminacies assuming independent and exponentially distributed activity durations. Neuman and Zimmermann [9] presented different heuristic and exact procedures for solving RCPSP with NPV criterion. Leyman and Vanhoucke [10] introduced a new schedule construction technique to improve the project NPV. Researches on RCPSP in early phase were done with the assumptions of complete information and deterministic environment. For a given project, a baseline schedule can be obtained by solving deterministic RCPSP. The deterministic RCPSP has been extensively studied and numerous exact and heuristic methods have been proposed to solve it $[11,12]$. However, the baseline schedule is vulnerable when being executed in indeterminate environment. In reality, there are considerable indeterminacies (accident, resource breakdown, unreliable deliveries, etc.), which may result in an infeasible baseline schedule. Hence, it is necessary to consider indeterminate factors when solving a project scheduling problem.

Recently, Fathallahi and Najafi [13] studied fuzzy RCPSP with NPV criterion. In the fuzzy set theory, the decision can be estimated by experts based on their experiences and professional judgments. However, fuzzy set theory may lead to counterintuitive results 
[14]. Besides, the stochastic resource-constrained project scheduling problem (SRCPSP), the main context of stochastic project scheduling, is characterized by random activity durations and scheduling policies. Activity durations are assumed to be random variables and a scheduling policy is used to decide which activities to be started at decision points (the starting time of project and the finishing times of activities) [15]. Generally, the SRCPSP aims at minimizing the expected makespan by making a limited set of decisions during project execution. Tsai and Gemmill [16] proposed tabu search for both deterministic and stochastic RCPSP. For more details about SRCPSP, readers may refer to $[12,17,18]$.

In SRCPSP, activity durations are represented by random variables. The assumption is reasonable when there are enough historical data to estimate variables' probability distributions precisely. However, in a project, it is difficult to get enough historical data for activities seldom or never executed. This situation is common in consideration of the uniqueness of a project. Therefore, new ways instead of probability distribution are needed to describe such variables. In this case, uncertainty theory, initiated by Liu [14], was founded to rationally deal with belief degrees, which inspired a new method of describing indeterministic phenomena. For now, the new theory has been successfully applied to varieties of fields, such as, option pricing problem [19-21], stock problem [22], production control problem [23], shortest path problem [24], etc. Ke et al. [25] researched project scheduling problem in the environment with uncertainty and randomness. For more details about uncertain project scheduling problem, readers may refer to [26, 27].

In this paper, we build three NPV models for URCPSPDC additionally considering a due date constraint.

\section{Preliminaries}

Uncertainty theory, as a branch of axiomatic mathematics, has been well developed in many aspects in reality. In this section, some concepts and theorems of uncertainty theory are introduced to lay the foundation for URCPSPDC modeling.

Let $\Gamma$ be a nonempty set, $\mathcal{L}$ a $\sigma$-algebra over $\Gamma$, and each element $\Lambda$ in $\mathcal{L}$ is named as an event. Uncertain measure $\mathcal{M}$, initiated by Liu [14] and perfected by Liu [28], is a function from $\mathcal{L}$ to $[10,1]$. It is defined over the following four axioms.

Axiom 1 (Normality Axiom) $\mathcal{M}\{\Gamma\}=1$.

Axiom 2 (Duality Axiom) $\mathcal{M}\{\Lambda\}+\mathcal{M}\left\{\Lambda^{c}\right\}=1$ for any event $\Lambda$.

Axiom 3 (Subadditivity Axiom) For every countable sequence of events $\left\{\Lambda_{i}\right\}, i=$ $1,2, \cdots$, we have:

$$
\mathcal{M}\left\{\bigcup_{i=1}^{\infty} \Lambda_{i}\right\} \leq \sum_{i=1}^{\infty} \mathcal{M}\left\{\Lambda_{i}\right\} .
$$

Axiom 4 (Product Measure Axiom) Let $\left(\Gamma_{k}, \mathcal{L}_{k}, \mathcal{M}_{k}\right)$ be uncertainty spaces for $k=$ $1,2, \cdots$ The product uncertain measure $\mathcal{M}$ is an uncertain measure satisfying

$$
\mathcal{M}\left\{\prod_{k=1}^{\infty} \Lambda_{k}\right\}=\bigwedge_{k=1}^{\infty} \mathcal{M}_{k}\left\{\Lambda_{k}\right\}
$$

where $\Lambda_{k}$ are arbitrarily chosen events from $\mathcal{L}_{k}$ for $k=1,2, \cdots$, respectively. 
Definition $1 \mathrm{Liu}$ [14] An uncertain variable is a measurable function $\xi$ from an uncertainty space $(\Gamma, \mathcal{L}, \mathcal{M})$ to the set of real numbers, i.e., for any Borel set $B$ of real numbers, the set

$\{\xi \in B\}=\{\gamma \in \Gamma \mid \xi(\gamma) \in B\}$

is an event.

The uncertainty distribution is indispensable to establish practical uncertain optimization models.

Definition 2 Liu [14] The uncertainty distribution $\Phi$ of an uncertain variable $\xi$ is defined by

$$
\Phi(x)=\mathcal{M}\{\xi \leq x\}
$$

for any real number $x$.

An uncertainty distribution $\Phi$ is confirmed to be regular if its inverse function $\Phi^{-1}(\alpha)$ exists uniquely for each $\alpha \in[0,1]$.

Definition 3 Liu [14] Let $\xi$ be an uncertain variable. The expected value of $\xi$ is defined by

$$
E[\xi]=\int_{0}^{+\infty} \mathcal{M}\{\xi \geq r\} \mathrm{d} r-\int_{-\infty}^{0} \mathcal{M}\{\xi \leq r\} \mathrm{d} r
$$

provided that at least one of the above two integrals is finite.

Lemma 1 Liu [28] Let $\xi$ be an uncertain variable with uncertainty distribution $\Phi$. If the expected value exists, then

$$
E[\xi]=\int_{0}^{+\infty}(1-\Phi(x)) \mathrm{d} x-\int_{-\infty}^{0} \Phi(x) \mathrm{d} x .
$$

Lemma 2 Liu [28] Let $\xi$ be an uncertain variable with regular uncertainty distribution $\Phi$. If the expected value exists, then

$$
E[\xi]=\int_{0}^{1} \Phi^{-1}(\alpha) \mathrm{d} \alpha
$$

Lemma 3 Liu [28] Let $\xi_{1}, \xi_{2}, \cdots, \xi_{n}$ be independent uncertain variables with regular uncertainty distributions $\Phi_{1}, \Phi_{2}, \cdots, \Phi_{n}$, respectively. A function $f\left(x_{1}, x_{2}, \cdots, x_{n}\right)$ is strictly increasing with respect to $x_{1}, x_{2}, \cdots, x_{m}$ and strictly decreasing with respect to $x_{m+1}, x_{m+2}, \cdots, x_{n}$. Then $\xi=f\left(\xi_{1}, \xi_{2}, \cdots, \xi_{n}\right)$ is an uncertain variable with inverse uncertainty distribution

$$
\Psi^{-1}(\alpha)=f\left(\Phi_{1}^{-1}(\alpha), \cdots, \Phi_{m}^{-1}(\alpha), \Phi_{m+1}^{-1}(1-\alpha), \cdots, \Phi_{n}^{-1}(1-\alpha)\right) .
$$

\section{NPV Models}

\section{Problem Description}

A project containing $n$ activities can be described by an activity-on-the-node network $G(N, A)$. The set of nodes $N=\{1,2, \cdots, n+2\}$ represents activities, and the set of $\operatorname{arcs} A$ denotes finish-start, zero-lag precedence relations between activities. Activities 1 and $n+$ 
2 do not consume time and resources, and only signify project starting point and finishing point, respectively. Specially, durations of all activities in URCPSPDC are represented by an uncertain vector $\boldsymbol{d}=\left\{0, \tilde{d}_{2}, \cdots, \tilde{d}_{n+1}, 0\right\}$ and the start times of all activities are denoted as a vector $\boldsymbol{s}=\left\{0, s_{2}, \cdots, s_{n+1}, s_{n+2}\right\}$. Therefore, the completion time of $i$ th activity $f_{i}$ can be calculated as $s_{i}+\tilde{d}_{i}$. Moreover, there are totally $K$ types of renewable resources and each of them has a constant availability $a_{k}, k=1,2, \cdots, K$. Besides, when activity $i$ is completed, a cash flow $C_{i}^{F}$ will be received accordingly and we discount it to the project staring time with a discount rate $r \in(0,1]$. In this paper, we additionally take a due date $\delta$ into consideration.

The URCPSPDC aims at maximizing the NPV of a whole project with uncertain activity durations meanwhile satisfying the deadline constraint. Solving URCPSPDC is a dynamic decision process. The decision maker decides to start which feasible activity at each decision point, including project starting time and activity finishing times. In the decision process, the decision maker can only utilize partial information which appears before his decision point.

With a given activity list $\pi$, an executing order of activities, the completion time of activity $i$ can be calculated as follows:

$$
f_{i}(\pi, \boldsymbol{d})=s_{i}(\pi, \boldsymbol{d})+\tilde{d}_{i}
$$

Without resource constraint, the starting time of activity $i$ can be computed considering precedence relationship as follows:

$$
s_{i}(\pi, \boldsymbol{d})=\max _{(j, i) \in A} f_{j}(\pi, \boldsymbol{d}) .
$$

However, the formula cannot always hold with resource constraint. Some activity is feasible in precedence relationship logic if all of its predecessors have been finished. In such a case, it can be sometimes infeasible for lack of available resources. In other words, an activity has predecessors in precedence relationship logic as well as in resource logic. To produce feasible schedule effectively, resource flow network was presented by Artigues and Roubellat [29]. If there is a resource flow, extra relation is added into the original network between activities without precedence relationship. Thus an extended precedence relationship set $A^{*}$ is developed. Combined with the side constraint proposed by Ma et al. [30], the starting time of activity $i$ can be calculated by

$$
s_{i}(\pi, \boldsymbol{d})=\max _{\pi_{m}<\pi_{i}} s_{m}(\pi, \boldsymbol{d}) \vee \max _{(j, i) \in A^{*}} f_{j}(\pi, \boldsymbol{d})
$$

where $\pi_{i}$ is the position of activity $i$ in activity list $\pi$.

Therefore, $s_{i}(\pi, \boldsymbol{d})$ is strictly increasing with respect to $\boldsymbol{d}$, then $s_{i}(\pi, \boldsymbol{d})+\tilde{d}_{i}$ is also strictly increasing with respect to $\boldsymbol{d}$, and $N p v(\pi, \boldsymbol{d})=\sum_{i=2}^{n+1} C_{i}^{F} r_{i}$ must be strictly decreasing with respect to $\boldsymbol{d}$ and $\boldsymbol{s}$. Thus, we can have 
Theorem 1 Provided that the duration of activity $i$ has a regular distribution $\Phi_{i}(x)$ and an inverse uncertainty distribution $\Phi_{i}^{-1}(\alpha), s_{i}(\pi)$ has an inverse uncertainty distribution $\Psi_{i}^{-1}(\pi, \alpha)$, and $N p v(\pi)$ has an inverse uncertainty distribution $\Upsilon^{-1}(\pi, \alpha), \alpha \in(0,1]$, we can get

$$
\begin{aligned}
& \Psi_{n+2}^{-1}(\pi, \alpha)=\max _{\pi_{m}<\pi_{n+2}} \Psi_{m}^{-1}(\pi, \alpha) \vee \max _{(j, n+2) \in A^{*}}\left(\Psi_{j}^{-1}(\pi, \alpha)+\Phi_{j}^{-1}(\alpha)\right), \\
& \Upsilon^{-1}(\pi, \alpha)=\sum_{i=2}^{n+1} C_{i}^{F} r^{\max _{m}<\pi_{i}} \Psi_{m}^{-1}(\pi, 1-\alpha) \vee \max _{(j, i) \in A^{*}}\left(\Psi_{j}^{-1}(\pi, 1-\alpha)+\Phi_{j}^{-1}(1-\alpha)\right)+\Psi_{i}^{-1}(\pi, 1-\alpha)
\end{aligned} .
$$

Theorem 2 The expected makespan and the expected NPV can be obtained as follows:

$$
E\left[s_{n+2}\right]=\int_{0}^{1} \Psi_{n+2}^{-1}(\pi, \alpha) \mathrm{d} \alpha, \quad E[N p \nu]=\int_{0}^{1} \Upsilon^{-1}(\pi, \alpha) \mathrm{d} \alpha .
$$

\section{Chance-Constrained Model}

The chance-constrained model applies chance-constrained programming [31] to maximize the NPV of cash flow received on each activity's completion time. For risk-averse decision makers, this model realizes the maximization of NPV with a relatively high belief degree. Meanwhile, a due date constraint is also taken into account. In other words, we aim to obtain a financial optimal solution with a due date constraint. The model is proposed as follows:

$$
\left\{\begin{array}{l}
\max \overline{N p v} \\
\text { subject to: } \\
\mathcal{M}\left\{\sum_{i=2}^{n+1} C_{i}^{F} r^{s_{i}+\tilde{d}_{i}} \geq \overline{N p \nu}\right\} \geq \alpha \\
\mathcal{M}\left\{s_{n+2} \leq \delta\right\} \geq \beta
\end{array}\right.
$$

where $\alpha, \beta$ are belief degrees.

In the above model, the objective function is to maximize the NPV with belief degree $\alpha$, enforced in the first constraint. The second constraint ensures the makespan cannot exceed the deadline with belief degree $\beta$.

Since $\mathcal{M}\{N p v \geq \overline{N p v}\} \geq \alpha$ can be transformed as $1-\Upsilon(\pi, \overline{N p v}) \geq \alpha$, we have $\Upsilon^{-1}(\pi, 1-\alpha) \geq \overline{N p \nu}$. Therefore, the objective value of $\overline{N p \nu}$ can be calculated as follows:

$$
\Upsilon^{-1}(\pi, 1-\alpha)=\sum_{i=2}^{n+1} C_{i}^{F} r^{\max _{m}<\pi_{i}} \Psi_{m}^{-1}(\pi, \alpha) \vee \max _{(j, i) \in A^{*}}\left(\Psi_{j}^{-1}(\pi, \alpha)+\Phi_{j}^{-1}(\alpha)\right)+\Psi_{i}^{-1}(\pi, \alpha) .
$$

Accordingly, the uncertain model can be transformed as follows:

$$
\left\{\begin{array}{l}
\max \Upsilon^{-1}(\pi, 1-\alpha) \\
\text { subject to: } \\
\Psi_{n+2}^{-1}(\pi, \beta) \leq \delta
\end{array}\right.
$$

\section{Expected Value Model}

The expected value model aims at maximizing the expected NPV of cash flow received on each activity's completion time. The model is proposed as follows:

$$
\left\{\begin{array}{l}
\max E[N p v] \\
\text { subject to: } \\
E\left[s_{n+2}\right] \leq \delta .
\end{array}\right.
$$


The equivalent form of the uncertain model is shown as follows:

$$
\left\{\begin{array}{l}
\max \int_{0}^{1} \Upsilon^{-1}(\pi, \alpha) \mathrm{d} \alpha \\
\text { subject to: } \\
\int_{0}^{1} \Psi_{n+2}^{-1}(\pi, \alpha) \mathrm{d} \alpha \leq \delta
\end{array}\right.
$$

With the crisp-form of the expected value model, 99-method can be applied to make approximation of the expected NPV:

$$
\left\{\begin{array}{l}
\max \sum_{m=1}^{99} \Upsilon^{-1}(\pi, m / 100) / 99 \\
\text { subject to: } \\
\sum_{m=1}^{99} \Psi_{n+2}^{-1}(\pi, m / 100) / 99 \leq \delta
\end{array}\right.
$$

\section{Chance Maximization Model}

Dependent-chance programming (DCP), initiated by Liu [32], is to optimize the chance of a certain event and we apply DCP to solve the project scheduling problem. Readers who are interested in DCP may refer to Liu and Iwamura [33] and Liu [34-36]. In this paper, the goal is given in advance as that the uncertainty of the NPV overrunning the goal should be as large as possible. The constraint is that the belief degree of finishing the project before the due date should be larger than or equal to a predetermined confidence level $\beta$. Hence, we can build the following chance maximization model:

$$
\left\{\begin{array}{l}
\max \mathcal{M}\{N p v \geq \overline{N p v}\} \\
\text { subject to: } \\
\mathcal{M}\left\{s_{n+2} \leq \delta\right\} \geq \beta
\end{array}\right.
$$

where $\beta$ is a belief degree.

The equivalent form of the uncertain model is shown as follows:

$$
\left\{\begin{array}{l}
\max (1-\alpha) \\
\text { subject to: } \\
\Upsilon^{-1}(\pi, 1-\alpha) \geq \overline{N p v} \\
\Psi_{n+2}^{-1}(\pi, \beta) \leq \delta
\end{array}\right.
$$

With the crisp-form of the chance maximization model, 99-method can be applied to make approximation of the uncertainty:

$$
\left\{\begin{array}{l}
\max 1-m / 100 \\
\text { subject to: } \\
\Upsilon^{-1}(\pi, 1-m / 100) \geq \overline{N p v} \\
\Psi_{n+2}^{-1}(\pi, \beta) \leq \delta
\end{array}\right.
$$

\section{Revised EDA}

Since deterministic RCPSP is NP-hard in the strong sense, URCPSPDC, an extension of RCPSP, needs to be solved by heuristic or meta-heuristic algorithm. In this section, a revised intelligent heuristic algorithm is designed by applying NPV criterion to uncertain 
serial schedule generation scheme (US-SGS) in EDA. For more details about EDA for RCPSP, readers may refer to $[4,5]$.

\section{Revised Uncertain Serial Schedule Generation Scheme}

As discussed by Kolish and Hartmann [37], there are several types of feasible solution representations for project scheduling. A former work by Ma et al. [30] designed an uncertain serial SGS (US-SGS) for uncertain RCPSP. For URCPSPDC in this paper, we choose the solution representation activity list $\pi$, which represents the executing order of activities. The US-SGS can be described as follows.

\section{Chance-Constrained Model}

0:Given a network $G(N, A) . \pi:=$ activity list; $\boldsymbol{d}:=$ duration time; $\boldsymbol{W}:=$ underway activity set; $\boldsymbol{U}:=$ unscheduled activity set; $\boldsymbol{s}:=$ starting time; $\boldsymbol{F}:=$ finishing time; $T:=$ time point; $n:=$ number of the activities; $N p v:=$ net present value.

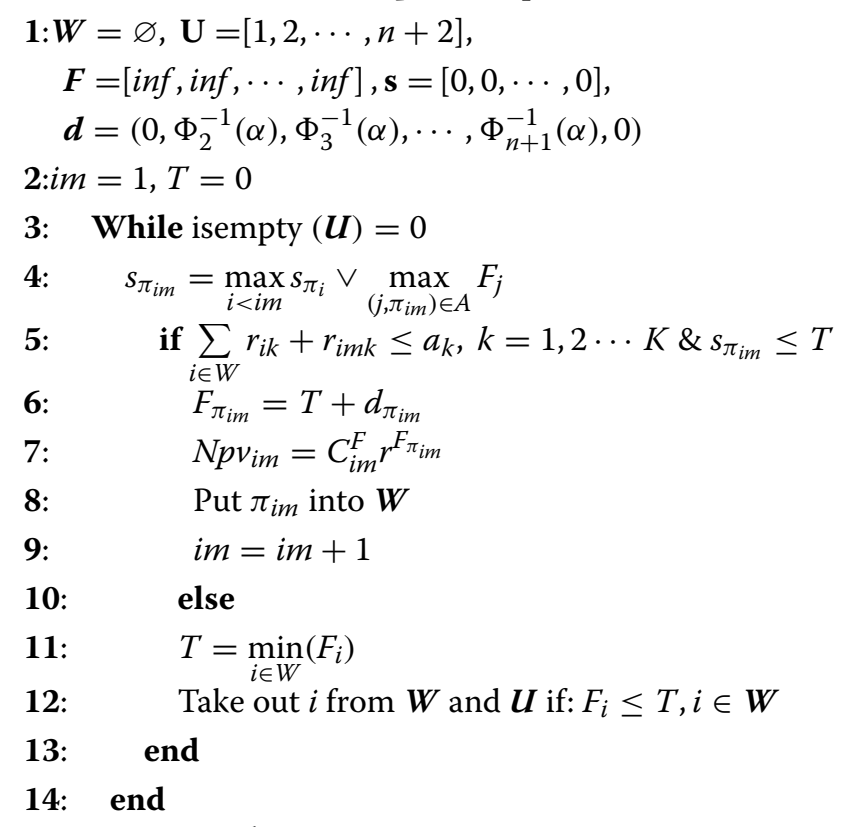

\section{5:Return $\sum_{i m=2}^{n+1} N p v_{i m}, s_{n+2}$.}

It is worth mentioning that a realized makespan with confidence level $\beta$ must be subject to the due date constraint and if not, the corresponding activity list has to be abandoned.

\section{Expected Value Model}

Compared with the chance-constrained model, the expected value model generates $d_{1}^{m}, d_{2}^{m}, \ldots, d_{n+2}^{m}$ according to the uncertainty distributions of activities' durations $\Phi_{1}, \Phi_{2}, \ldots, \Phi_{n+2}$. Denote $\boldsymbol{d}^{\boldsymbol{m}}=\left(0, \Phi_{2}^{-1}(m / 100), \Phi_{3}^{-1}(m / 100), \ldots, \Phi_{n+1}^{-1}\right.$ $(m / 100), 0), m=1,2, \ldots, 99$, respectively. Next, by using the decoding scheme concerned above, $s_{n+2}^{m}$ and $N p v^{m}$ can be obtained. Finally we can get $E[N p v]=\sum_{m=1}^{99} N p v^{m} / 99$. Note that an expected makespan $E\left[s_{n+2}\right]=\sum_{m=1}^{99} s_{n+2}^{m} / 99$ must be subject to the due date constraint and if not, the corresponding activity list has to be abandoned.

\section{Chance Maximization Model}

Compared with the above two models, for each activity list $\pi$, this model first obtains a realized makespan with confidence level $\beta$ and a series of $N p v^{m}, m=1,2, \ldots, 99$. In 
the condition of $N p v^{m} \geq \overline{N p v}$, we choose the largest $m$ among the feasible solution set. Note that the realized makespan with confidence level $\beta$ must be subject to the due date constraint and if not, the corresponding activity list has to be abandoned.

\section{Revised EDA}

In this section, the US-SGS is embedded into EDA. By this step, we employ the US-SGS to decode the activity lists and to approximate the fitness of each solution by uncertain simulation based on 99-method, initiated by liu [28] and successfully applied in project scheduling by Zhang and Chen [26]. In contrast to genetic algorithm (GA), EDA does not directly generate new solutions by crossover of parent solutions and mutations but by sampling from a probability distribution. The latter depicts the features of a selected set of feasible solutions of the problem. The outline of EDA is presented in Fig. 1.

In this paper, to revise the EDA, here are the steps. First, $N P$ solutions are generated according to the initial probability matrix as the initial population and we update probability matrix according to the initial population. Each solution is an activity list, where one activity can only be assigned if all of its predecessors have been finished. Second, the US-SGS is utilized to generate schedules according to activity lists, to filter infeasible solutions when realized makespan exceeds the deadline and to evaluate each solution. After

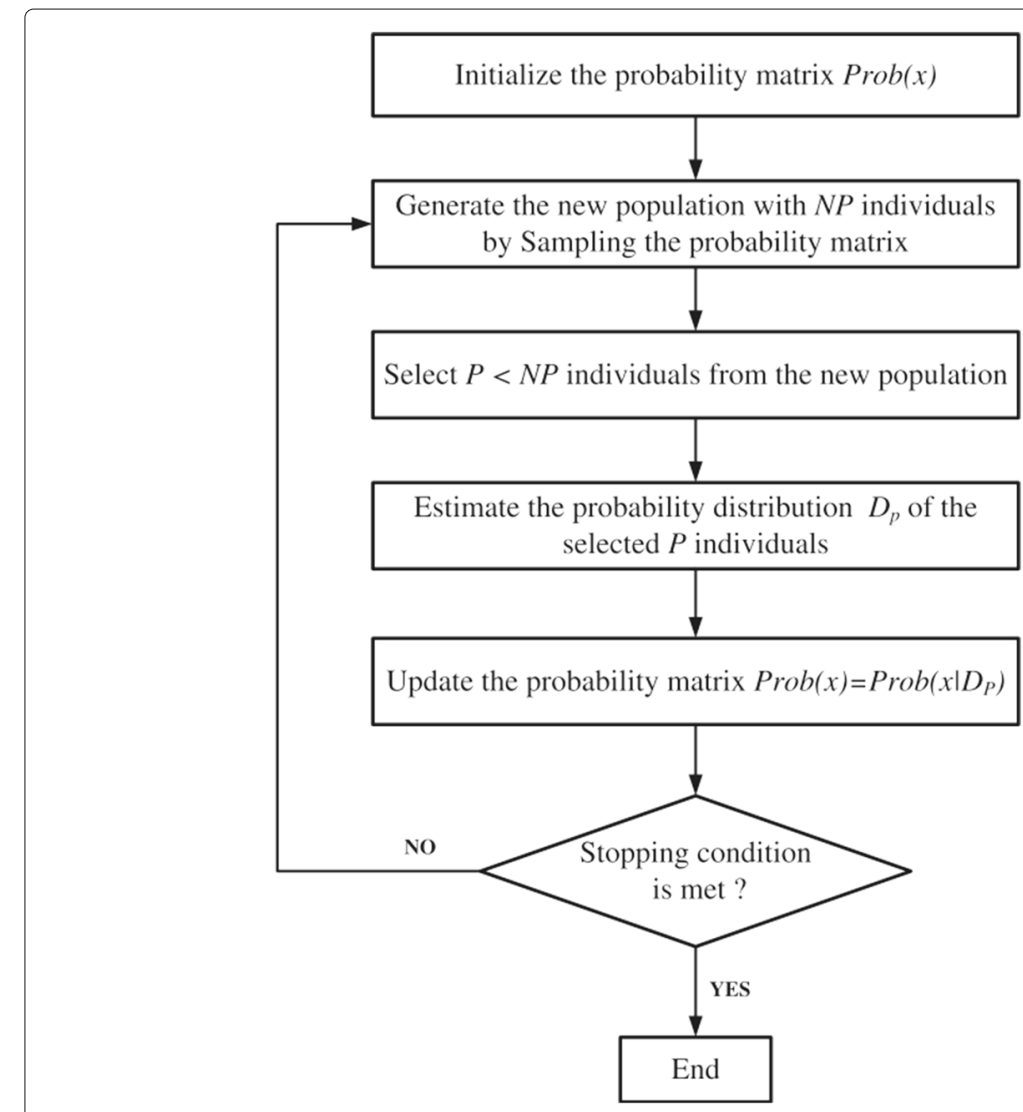

Fig. 1 The outline of EDA 
evaluating the population, $P<N P$ best individuals are selected from the population to form the elite set. And, the elite set is chosen to update probability matrix. Then, the new probability matrix is employed to sample population of the next generation. After a certain number of generations, the solution with best fitness value is reported as quasi-optimal solution.

\section{Numerical Examples}

A project with 30 activities and 4 renewable resources is taken as an example in this section. Some specific information about the project is shown in Table 1, including activity durations, resource requirements, successors, and cash flows. All the activity durations are assumed to be uncertain variables and described by uncertainty distributions estimated by experts.

Accordingly, the project structure is depicted in Fig. 2.

Table 1 Project information

\begin{tabular}{|c|c|c|c|c|c|c|c|}
\hline Activity & Duration & $R_{1}$ & $R_{2}$ & $R_{3}$ & $R_{4}$ & Successors & Cash flows \\
\hline 1 & 0 & 0 & 0 & 0 & 0 & $2,3,4$ & 0 \\
\hline 2 & $\mathcal{Z}(5,7,8)$ & 4 & 0 & 0 & 0 & $8,10,13$ & 105 \\
\hline 3 & $\mathcal{Z}(7,9,10)$ & 10 & 0 & 0 & 0 & $5,9,19$ & 54 \\
\hline 4 & $\mathcal{L}(1,3)$ & 0 & 0 & 0 & 3 & $6,16,17$ & 149 \\
\hline 5 & $\mathcal{Z}(1,3,4)$ & 3 & 0 & 0 & 0 & $10,18,31$ & 112 \\
\hline 6 & $\mathcal{L}(8,10)$ & 0 & 0 & 0 & 8 & 7,22 & 128 \\
\hline 7 & $\mathcal{Z}(7,8,10)$ & 4 & 0 & 0 & 0 & 28 & 87 \\
\hline 8 & $\mathcal{Z}(1,3,4)$ & 0 & 1 & 0 & 0 & 11,12 & 85 \\
\hline 9 & $\mathcal{L}(1,3)$ & 6 & 0 & 0 & 0 & 14,27 & 115 \\
\hline 10 & $\mathcal{Z}(8,10,11)$ & 0 & 0 & 0 & 1 & 30 & 125 \\
\hline 11 & $\mathcal{L}(7,10)$ & 0 & 5 & 0 & 0 & 24 & 143 \\
\hline 12 & $\mathcal{Z}(8,10,11)$ & 0 & 7 & 0 & 0 & 15,21 & 52 \\
\hline 13 & $\mathcal{Z}(1,3,4)$ & 4 & 0 & 0 & 0 & 17 & 80 \\
\hline 14 & $\mathcal{L}(1,3)$ & 0 & 8 & 0 & 0 & 20 & 76 \\
\hline 15 & $\mathcal{Z}(3,5,6)$ & 3 & 0 & 0 & 0 & 30 & 86 \\
\hline 16 & $\mathcal{L}(2,4)$ & 0 & 0 & 0 & 5 & 25 & 126 \\
\hline 17 & $\mathcal{L}(7,11)$ & 0 & 0 & 0 & 8 & 21 & 146 \\
\hline 18 & $\mathcal{Z}(6,8,9)$ & 0 & 0 & 0 & 7 & 29 & 121 \\
\hline 19 & $\mathcal{Z}(2,4,5)$ & 0 & 1 & 0 & 0 & $20,23,24$ & 93 \\
\hline 20 & $\mathcal{Z}(7,10,11)$ & 0 & 10 & 0 & 0 & 21 & 140 \\
\hline 21 & $\mathcal{L}(4,6)$ & 0 & 0 & 0 & 6 & 28 & 68 \\
\hline 22 & $\mathcal{Z}(2,4,5)$ & 2 & 0 & 0 & 0 & 26 & 69 \\
\hline 23 & $\mathcal{L}(3,5)$ & 3 & 0 & 0 & 0 & 26 & 52 \\
\hline 24 & $\mathcal{Z}(3,5,6)$ & 0 & 9 & 0 & 0 & 25,29 & 123 \\
\hline 25 & $\mathcal{L}(6,8)$ & 4 & 0 & 0 & 0 & 30 & 78 \\
\hline 26 & $\mathcal{Z}(4,5,7)$ & 0 & 0 & 4 & 0 & 28 & 129 \\
\hline 27 & $\mathcal{L}(1,3)$ & 0 & 0 & 0 & 7 & 31 & 137 \\
\hline 28 & $\mathcal{Z}(2,3,5)$ & 0 & 8 & 0 & 0 & 31 & 77 \\
\hline 29 & $\mathcal{Z}(1,2,4)$ & 0 & 7 & 0 & 0 & 32 & 125 \\
\hline 30 & $\mathcal{Z}(5,6,8)$ & 0 & 7 & 0 & 0 & 32 & 146 \\
\hline 31 & $\mathcal{Z}(4,6,7)$ & 0 & 0 & 2 & 0 & 32 & 58 \\
\hline 32 & 0 & 0 & 0 & 0 & 0 & & 0 \\
\hline
\end{tabular}




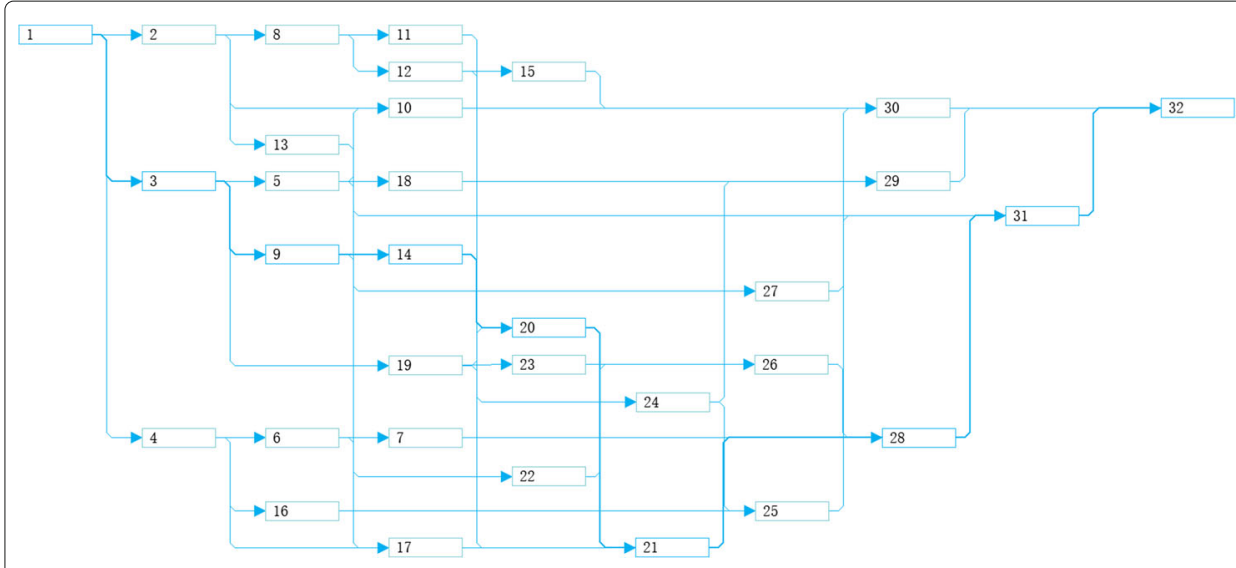

Fig. 2 The network of the project

\section{Chance-Constrained Model}

Suppose that the discount rate is 0.99 and the financial officer wants to realize the objective value with confidence level 0.9 while the project manager prefers to determine whether the makespan is acceptable with confidence level 0.85 . The chance-constrained model can be rewritten as:

$$
\left\{\begin{array}{l}
\max \overline{N p v} \\
\text { subject to: } \\
\mathcal{M}\left\{\sum_{i=2}^{n+1} C_{i}^{F} * 0.99^{s_{i}+\tilde{d}_{i}} \geq \overline{N p v}\right\} \geq 0.9 \\
\mathcal{M}\left\{s_{32} \leq 90\right\} \geq 0.85
\end{array}\right.
$$

In order to clarify the expression of formulas, we denote an uncertain duration vector with a belief degree $m / 100$ as $\boldsymbol{d}^{m}=\left(0, \Phi_{2}^{-1}(m / 100), \Phi_{3}^{-1}(m / 100), \ldots, \Phi_{n+1}^{-1}\right.$ $(m / 100), 0), m=1,2, \ldots, 99$. Then, $s_{n+2}^{m}$ and $N p v^{m}$ represent a makespan and an NPV with a belief degree $m / 100$, respectively. Accordingly, the uncertain model can be transformed as follows:

$$
\left\{\begin{array}{l}
\max \sum_{i=2}^{n+1} C_{i}^{F} * 0.99^{s_{i}^{90}+\tilde{d}_{i}}{ }^{90} \\
\text { subject to: } \\
s_{32}^{85} \leq 90
\end{array}\right.
$$

In this paper, specially, the NPV is changeable according to the discount rate $r$ and belief degrees $(\alpha, \beta)$. To simplify the problem, we take $(\alpha, \beta)$ the same values $(0.05,0.05)$, $(0.15,0.15), \ldots,(0.95,0.95)$ and consider the discount rate $r$ as 0.99 . The revised EDA runs 1000 generations with 10 times for 10 groups, respectively. Then, we list the best solution of all 10 times for each group. The quasi-optimal solutions and NPVs are provided in Table 2.

The result may help risk-averse decision makers from the following two aspects: First, a financial officer can arrange the capital operation plan with NPV based on a project manager's makespan prediction according to their own belief degrees;. Second, a given belief degree corresponds with an optimal schedule.

To conclude, given a fixed discount rate, a higher belief degree corresponds with a lower realized NPV and the optimal solution varies according to different belief degrees. Also, 
Table 2 The quasi-optimal solutions for chance-constrained model

\begin{tabular}{llll}
\hline$(\alpha, \beta)$ & Quasi-optimal schedule & NPV & Makespan \\
\hline$(0.05,0.05)$ & $1,2,8,4,3,6,12,9,5,11,13,22,16,18,7,15,19,23$, & 3421.4 & 47.75 \\
$(0.15,0.15)$ & $24,10,14,27,29,25,20,17,26,30,21,28,31,32$ & & \\
& $1,4,2,8,6,3,11,12,13,19,16,5,22,18,24,9,23,27$, & 3342.4 & \\
$(0.25,0.25)$ & $14,15,17,10,20,25,30,26,29,7,21,28,31,32$ & & 54 \\
$(0.35,0.35)$ & $1,4,2,8,3,6,11,12,9,13,14,5,7,27,16,19,23,24$, & 3311.1 & 60.2 \\
$(0.45,0.45)$ & $18,15,10,20,17,22,25,26,30,29,21,28,31,32$ & & \\
& $1,3,4,19,9,6,5,2,14,20,13,10,23,8,27,22,7,26$, & 3255.8 & \\
$(0.55,0.55)$ & $18,11,12,16,24,17,21,25,15,28,31,29,30,32$ & & 61.4 \\
$(0.65,0.65)$ & $1,4,2,8,6,3,11,12,19,16,9,5,22,18,10,14,13,20$, & 3206.6 & \\
$(0.75,0.75)$ & $23,7,17,26,27,24,21,15,25,28,30,31,29,32$ & & \\
& $1,2,4,8,3,6,12,11,7,9,16,5,14,10,19,18,22,23$, & 3199.5 & \\
$(0.85,0.85)$ & $13,24,27,25,26,20,15,17,30,21,28,31,29,32$ & & \\
$(0.95,0.95)$ & $1,4,2,8,6,3,11,12,19,5,9,16,22,10,18,24,14$, & 3163.5 & \\
& $13,20,23,7,27,17,15,25,21,30,26,29,28,31,32$ & & \\
& $1,4,3,2,6,9,19,23,5,13,14,16,8,27,20,22,26$, & 3093.5 & \\
\hline
\end{tabular}

an optimal NPV solution is not necessary to reach a best makespan. Therefore, for riskaverse decision makers, it is considerable to choose applicable belief degrees to solve this problem.

\section{Expected Value Model}

Suppose that the discount rate is 0.99 . Then the expected value model can be rewritten as:

$$
\left\{\begin{array}{l}
\max E\left[\sum_{i=2}^{n+1} C_{i}^{F} * 0.99^{s_{i}+\tilde{d}_{i}}\right] \\
\text { subject to: } \\
E\left[s_{32}\right] \leq 90
\end{array}\right.
$$

With the crisp-form of the expected value model, 99-method can be applied to make approximation of the expected NPV:

$$
\left\{\begin{array}{l}
\max \sum_{m=1}^{99}\left(\sum_{i=2}^{n+1} C_{i}^{F} * 0.99^{s_{i}^{m}+\tilde{d}_{i}^{m}}\right) / 99 \\
\text { subject to: } \\
\sum_{m=1}^{99} s_{32}^{m} / 99 \leq 90 .
\end{array}\right.
$$

Then, we run 10 times with a discount rate 0.99 for three groups, respectively. The three groups are different from generation and popsize. The 10 best quasi-optimal solutions and NPVs are provided in Table 3.

According to Table 3, we can get the best NPV, the worst NPV, the average NPV, and we can calculate the error as shown in Table 4. The result reveals that the deviation denoted by percent error does not exceed 5\%, which implies the effectiveness of the algorithm integrating uncertain simulations. The result may help financial officers who are riskneutral arrange the capital operation plan with NPV. 
Table 3 The quasi-optimal solutions for expected value model

\begin{tabular}{|c|c|c|c|}
\hline Generation and popsize & Quasi-optimal schedule & E[NPV] & E[makespan] \\
\hline 150 and 30 & $\begin{array}{l}1,3,19,4,2,9,5,14,6,20,13,8,10,22,27,23,18 \\
12,11,17,15,16,7,21,24,29,26,28,31,25,30,32\end{array}$ & 3172 & 65 \\
\hline 500 and 30 & $\begin{array}{l}1,4,3,19,16,2,5,9,8,13,14,27,23,6,12,11,22,7 \\
18,10,15,26,24,20,25,17,30,21,28,31,29,32\end{array}$ & 3182.4 & 65.068 \\
\hline 500 and 30 & $\begin{array}{l}1,2,8,3,4,6,12,9,19,13,15,22,11,27,16,5,14,23 \\
17,7,26,20,21,28,18,31,24,10,29,25,30,32\end{array}$ & 3144.7 & 71.263 \\
\hline 500 and 30 & $\begin{array}{l}1,3,4,19,2,9,23,6,5,14,13,10,20,8,16,22,7,11 \\
27,12,18,17,24,15,21,29,26,25,28,30,31,32\end{array}$ & 3147.3 & 69.505 \\
\hline 150 and 50 & $\begin{array}{l}1,4,2,16,8,3,6,22,13,7,19,17,23,12,5,11,26,10 \\
9,14,20,27,24,25,15,21,28,31,18,30,29,32\end{array}$ & 3148.4 & 63.763 \\
\hline 150 and 50 & $\begin{array}{l}1,2,4,8,3,6,7,22,11,12,19,5,13,23,16,26,17,9 \\
24,10,14,20,18,25,15,29,21,28,27,31,30,32\end{array}$ & 3180.2 & 60.505 \\
\hline 150 and 50 & $\begin{array}{l}1,3,2,9,27,14,5,4,19,8,13,23,20,16,6,10,22,12 \\
17,11,7,26,21,28,15,24,18,25,31,30,29,32\end{array}$ & 3151.5 & 68.753 \\
\hline 150 and 50 & $\begin{array}{l}1,2,4,16,8,3,6,11,12,5,9,19,13,24,17,7,27,25 \\
14,10,20,22,18,15,23,26,21,29,30,28,31,32\end{array}$ & 3156.8 & 62.131 \\
\hline 150 and 50 & $\begin{array}{l}1,2,4,8,3,6,11,12,9,19,5,10,15,23,24,22,27,14 \\
20,7,13,26,16,17,25,21,28,30,18,29,31,32\end{array}$ & 3232 & 68.258 \\
\hline 150 and 50 & $\begin{array}{l}1,2,4,8,3,6,12,11,13,19,16,7,22,24,5,18,9,25 \\
14,15,29,27,17,23,20,10,21,30,26,28,31,32\end{array}$ & 3162.6 & 73.288 \\
\hline
\end{tabular}

\section{Chance Maximization Model}

Suppose that the discount rate is 0.99 and the financial officer wants to get the uncertainty at which the NPV exceeds 3000 while satisfying the constraint that the makespan is acceptable with confidence level 0.85 . The chance maximization model can be rewritten as follows:

$$
\left\{\begin{array}{l}
\max \mathcal{M}\left\{\sum_{i=2}^{n+1} C_{i}^{F} * 0.99^{s_{i}+\tilde{d}_{i}} \geq 3000\right\} \\
\text { subject to: } \\
\mathcal{M}\left\{s_{32} \leq 90\right\} \geq 0.85
\end{array}\right.
$$

With the crisp-form of the chance maximization model, 99-method can be applied as follows:

$$
\left\{\begin{array}{l}
\max \quad 1-m / 100 \\
\text { subject to: } \\
\sum_{i=2}^{n+1} C_{i}^{F} * 0.99^{s_{i}^{100-m}}+\tilde{d}_{i}^{100-m} \geq 3000 \\
s_{32}^{85} \leq 90 .
\end{array}\right.
$$

In this paper, we take discount rate as a fixed value 0.99 and test different values for $\overline{N p v}$. The revised EDA runs 1000 generations for five groups, respectively. The quasi-optimal solutions and belief degrees are provided in Table 5 .

Table 4 The effectiveness of the EDA

\begin{tabular}{lllll}
\hline Size & The best & The worst & The average & Error \\
\hline 30 & 3232 & 3077.8 & 3142.30 & $4.77 \%$ \\
\hline
\end{tabular}


Table 5 The quasi-optimal solutions for chance maximization model

\begin{tabular}{clll}
$\overline{\overline{N p v}}$ & Quasi-optimal schedule & Uncertainty & NPV \\
\hline 3000 & $1,4,2,16,8,3,6,22,13,9,19,11,5,7,27,23,14,17$, & 0.99 & 3021.7 \\
& $10,12,20,15,18,26,24,25,29,21,28,31,30,32$ & & 3104.6 \\
3100 & $1,2,4,8,3,6,12,22,5,19,9,11,10,23,14,18,24,26$, & 0.69 & 3203.7 \\
3200 & $13,16,20,7,15,17,21,28,25,27,30,31,29,32$ & & 3301.6 \\
3300 & $1,4,2,8,6,3,11,12,5,16,22,9,19,14,23,24,18,15$ & 0.46 & \\
& $25,27,26,7,13,17,20,21,29,10,28,31,30,32$ & & 3403.8 \\
3400 & $1,3,4,19,6,9,5,2,14,20,13,22,27,23,10,8,12,11$ & 0.27 & \\
& $18,26,7,16,24,15,17,25,21,30,28,31,29,32$ & & \\
& $1,2,4,16,8,3,6,11,12,19,5,15,9,10,24,23,14,18$, & & \\
\end{tabular}

According to Table 5, we can compare the results with the chance-constrained model and the two models validate each other. The result may help financial officers from the following two aspects. First, a financial officer can determine an uncertainty at which a given NPV value can be reached based on a project manager's makespan prediction according to a belief degree. Second, a given predetermined NPV corresponds with an optimal schedule.

To conclude, for financial officers, it is considerable to set applicable NPV goals and constraint belief degrees to solve this problem.

\section{Conclusion and Future Work}

In the real project, the environment for project execution is full of indeterminacies. Considering the uniqueness of a project, it is common that activities are seldom or never executed before. As a result, it is difficult to describe activity durations by probability distributions for lack of historical data. Also, fuzzy set theory may lead to counterintuitive results. This paper considers NPV of RCPSP with uncertain durations and a deadline constraint. To satisfy different demand of financial officers, three uncertain models are built. We utilize a special SGS for our problem called US-SGS and added it into EDA. Furthermore, some numerical examples are solved with our models and algorithms. We hope our work may provide financial criterion for financial officers. For future work, we believe that it is worthwhile to take other factors as uncertain variables such as discount rate and to apply NPV criterion to other uncertain types of project scheduling problems.

\section{Acknowledgements}

This work was supported by National Natural Science Foundation of China (No.71371141) and the Fundamental Research Funds for the Central Universities.

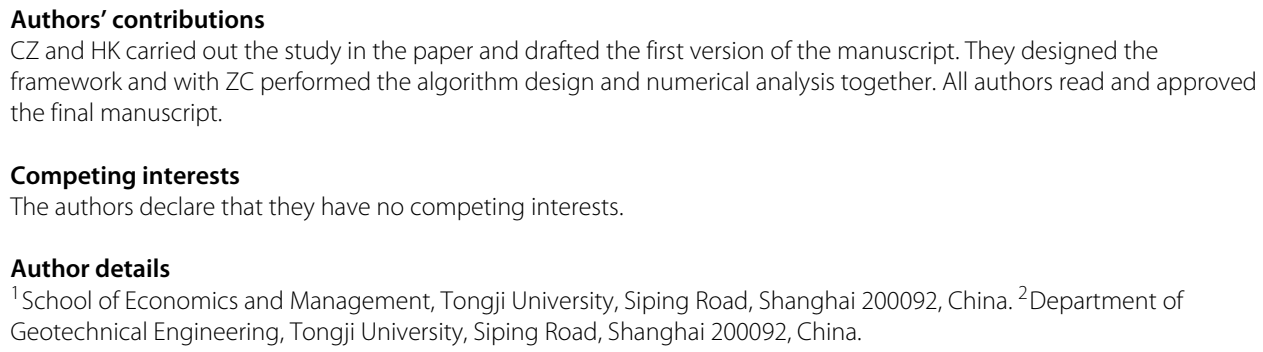




\section{References}

1. Herroelen, W, Demeulemeester, E, De Reyck, B: A classification scheme for project scheduling. In: Project Scheduling: Recent Models, Algorithms and Applications, pp. 1-26. Kluwer, Amsterdam, (1999)

2. Kolisch, R, Padman, R: An integrated survey of deterministic project scheduling. Omega. 29(3), 249-272 (2001)

3. Herroelen, WS, Van Dommelen, P, Demeulemeester, EL: Project network models with discounted cash flows: a guided tour through recent developments. Eur. J. Oper. Res. 100(1), 97-121 (1997)

4. Wang, L, Fang, C: An effective estimation of distribution algorithm for the multi-mode resource-constrained project scheduling problem. Comput. Oper. Res. 39(2), 449-460 (2012)

5. Fang, C, Kolisch, R, Wang, L, Mu, C: An estimation of distribution algorithm and new computational results for the stochastic resource-constrained project scheduling problem. Flex. Serv. Manuf. J. 27(4), 585-605 (2015)

6. Russell, A: Cash flows in networks. Manag. Sci. 16(5), 357-373 (1970)

7. Buss, AH, Rosenblatt, MJ: Activity delay in stochastic project networks. Oper. Res. 45(1), 126-139 (1997)

8. Sobel, MJ, Szmerekovsky, JG, Tilson, V: Scheduling projects with stochastic activity duration to maximize expected net present value. Eur. J. Oper. Res. 198(3), 697-705 (2009)

9. Neumann, K, Zimmermann, J: Procedures for resource leveling and net present value problems in project scheduling with general temporal and resource constraints. Eur. J. Oper. Res. 127(2), 425-443 (2000)

10. Leyman, P, Vanhoucke, M: A new scheduling technique for the resource-constrained project scheduling problem with discounted cash flows. Int. J. Prod. Res. 53(9), 2771-2786 (2015)

11. Demeulemeester, E, Herroelen, W: Project scheduling - a research handbook. Vol. 49 of International Series in Operations Research \& Management Science. Kluwer Academic Publishers, Boston (2002)

12. Ballestín, F: When it is worthwhile to work with the stochastic RCPSP. J. Sched. 10(3), 153-166 (2007)

13. Fathallahi, F, Najafi, A: A hybrid genetic algorithm to maximize net present value of project cash flows in resource-constrained project scheduling problem with fuzzy parameters. Scientia. Iranica. 23(4), 1893-1903 (2016)

14. Liu, B: Uncertainty Theory. 2nd edn. Springer, Berlin (2007)

15. Igelmund, G, Radermacher, FJ: Preselective strategies for the optimization of stochastic project networks under resource constraints. Networks. 13(1), 1-28 (1983)

16. Tsai, YW, Gemmill, DD: Using tabu search to schedule activities of stochastic resource-constrained projects. Eur. J. Oper. Res. 111(1), 129-141 (1998)

17. Ballestin, F, Leus, R: Resource-constrained project scheduling for timely project completion with stochastic activity durations. Prod. Oper. Manag. 18(4), 459-474 (2009)

18. Ashtiani, B, Leus, R, Aryanezhad, M-B: New competitive results for the stochastic resource-constrained project scheduling problem: exploring the benefits of pre-processing. J. Sched. 14(2), 157-171 (2011)

19. Chen, $X$ : American option pricing formula for uncertain financial market. Int. J. Oper. Res. 8(2), 32-37 (2011)

20. Peng, J, Yao, K: A new option pricing model for stocks in uncertainty markets. Int. J. Oper. Res. 8(2), 18-26 (2011)

21. Gao, Z, Wang, X, Ha, M: Multi-asset option pricing in an uncertain financial market with jump risk. J. Uncertain. Anal. Appl. 4(1), 1 (2016)

22. Bhattacharyya, R, Chatterjee, A, Kar, S: Uncertainty theory based multiple objective mean-entropy-skewness stock portfolio selection model with transaction costs. J. Uncertain. Anal. Appl. 1(1), 16 (2013)

23. Liu, B, Yao, K: Uncertain multilevel programming: algorithm and applications. Comput. Ind. Eng. 89, 235-240 (2015)

24. Zhang, Y, Liu, P, Yang, L, Gao, Y: A bi-objective model for uncertain multi-modal shortest path problems. J. Uncertain. Anal. Appl. 3(1), 8 (2015)

25. Ke, H, Liu, H, Tian, G: An uncertain random programming model for project scheduling problem. Int. J. Intell. Syst. 30(1), 66-79 (2015)

26. Zhang, X, Chen, $X$ : A new uncertain programming model for project scheduling problem. Inf. Int. Interdiscip. J. 15(10), 3901-3910 (2012)

27. Ke, H: Uncertain random time-cost trade-off problem. J. Uncertain. Anal. Appl. 2(1), 23 (2014)

28. Liu, B: Uncertainty Theory. 4th edn. Springer, Berlin (2010)

29. Artigues, C, Roubellat, F: A polynomial activity insertion algorithm in a multi-resource schedule with cumulative constraints and multiple modes. Eur. J. Oper. Res. 127(2), 297-316 (2000)

30. $\mathrm{Ma}, \mathrm{W}, \mathrm{Che}, \mathrm{Y}, \mathrm{Huang}, \mathrm{H}, \mathrm{Ke}, \mathrm{H}$ : Resource-constrained project scheduling problem with uncertain durations and renewable resources. Int. J. Mach. Learn. Cybernet. 7(4), 613-621 (2015)

31. Charnes, A, Cooper, WW: Chance-constrained programming. Manag. Sci. 6(1), 73-79 (1959)

32. Liu, B: Dependent-chance programming: a class of stochastic optimization. Comput. Math. Appl. 34(12), 89-104 (1997)

33. Liu, B, Iwamura, K: Modelling stochastic decision systems using dependent-chance programming. Eur. J. Oper. Res. 101(1), 193-203 (1997)

34. Liu, B: Uncertain programming. John Wiley \& Sons, Inc., New York (1999)

35. Liu, B: Uncertain programming: a unifying optimization theory in various uncertain environments. Appl. Math. Comput. 120(1), 227-234 (2001)

36. Liu, B: Theory and practice of uncertain programming. Springer, Heidelberg (2002)

37. Kolisch, R, Hartmann, S: Heuristic algorithms for the resource-constrained project scheduling problem: classification and computational analysis. In: Project Scheduling: Recent Models, Algorithms and Applications, pp. 147-178. Kluwer Academic Publishers, Netherlands, (1999) 